\title{
DISCI in Astrocytes Influences Adult Neurogenesis and Hippocampus-Dependent Behaviors in Mice
}

\author{
Chantelle E Terrillion ${ }^{1,8}$, Bagrat Abazyan ${ }^{1,8}$, Zhongxi Yang ${ }^{2,3,8}$, Joshua Crawford', Alexey V Shevelkin', \\ Yan Jouroukhin', Ki Hyun Yoo ${ }^{2}$, Chang Hoon Cho ${ }^{2}$, Robin Roychaudhuri ${ }^{4}$, Solomon H Snyder,4, \\ Mi-Hyeon Jang ${ }^{2,5,9}$ and Mikhail V Pletnikov ${ }^{1,4,6,7,9}$
}

'Department of Psychiatry and Behavioral Sciences, Johns Hopkins University School of Medicine, Baltimore, MD, USA; '2Department of Neurologic Surgery, Mayo Clinic College of Medicine, Rochester, MN, USA; 'Department of Neurosurgery, First Hospital of Jilin University, Changchun, China;

${ }^{4}$ Solomon H. Snyder Department of Neuroscience, Johns Hopkins University School of Medicine, Baltimore, MD, USA; ${ }^{5}$ Department of Biochemistry and Molecular Biology, Mayo Clinic College of Medicine, Rochester, MN, USA; ${ }^{6}$ Department of Molecular and Comparative Pathobiology, Johns Hopkins University School of Medicine, Baltimore, MD, USA; 7 Department of Molecular Microbiology and Immunology, Johns Hopkins Bloomberg School of Public Health, Baltimore, MD, USA

\begin{abstract}
The functional role of genetic variants in glia in the pathogenesis of psychiatric disorders remains poorly studied. Disrupted-In-Schizophrenia I (DISCI), a genetic risk factor implicated in major mental disorders, has been implicated in regulation of astrocyte functions. As both astrocytes and DISCI influence adult neurogenesis in the dentate gyrus (DG) of the hippocampus, we hypothesized that selective expression of dominant-negative C-terminus-truncated human DISCI (mutant DISCI) in astrocytes would affect adult hippocampal neurogenesis and hippocampus-dependent behaviors. A series of behavioral tests were performed in mice with or without expression of mutant DISCI in astrocytes during late postnatal development. In conjunction with behavioral tests, we evaluated adult neurogenesis, including neural progenitor proliferation and dendrite development of newbom neurons in the DG. The ameliorative effects of D-serine on mutant DISCI-associated behaviors and abnormal adult neurogenesis were also examined. Expression of mutant DISCI in astrocytes decreased neural progenitor proliferation and dendrite growth of newborn neurons, and produced elevated anxiety, attenuated social behaviors, and impaired hippocampus-dependent learning and memory. Chronic treatment with D-serine ameliorated the behavioral alterations and rescued abnormal adult neurogenesis in mutant DISCI mice. Our findings suggest that psychiatric genetic risk factors expressed in astrocytes could affect adult hippocampal neurogenesis and contribute to aspects of psychiatric disease through abnormal production of D-serine.
\end{abstract}

Neuropsychopharmacology (2017) 42, 2242-225I; doi:I0.1038/npp.2017.129; published online 19 July 2017

\section{INTRODUCTION}

Recent progress in psychiatric genetics has advanced our knowledge of how genetic variants can affect neurodevelopment and adult brain function (Sullivan et al, 2012). Unfortunately, our understanding of the underlying molecular mechanisms is limited to neurons, and little is known about the contributions of genetic risk factors within glia despite increasing evidence for their role in neurological and mental diseases (Kondziella et al, 2007; Schnieder and Dwork, 2011). Thus, we have recently begun studying the role(s) of Disrupted-In-Schizophrenia-1 (DISC1) in astrocytes (Ma et al, 2013).

* Correspondence: Professor MV Pletnikov, Johns Hopkins University School of Medicine, 600 North Wolfe Street, CMSC 8-121, Baltimore, MD 21287, USA, Tel: +4l0-502-3760, Fax: +4l0-614-0013, E-mail: mpletnik@jhmi.edu

${ }^{8}$ These authors contributed equally to this work.

${ }^{9}$ Co-senior authors.

Received 28 February 2017; revised 12 June 2017; accepted I5 June 2017; accepted article preview online 20 June 2017
DISC1 is a gene disrupted by the balanced (1:11) (q42.1; q14.3) translocation, segregating in the Scottish family with several major psychiatric disorders (Millar et al, 2000a). Although the DISC1 locus has not been reported in the latest genome-wide association studies (Schizophrenia Working Group of the Psychiatric Genomics, 2014), rare mutations of large effects contribute to mental disorders (Farrell et al, 2015) and are critical for uncovering the molecular pathobiology of psychiatric disease (Geschwind and Flint, 2015; Sullivan et al, 2012). It is in this context that we consider DISC1 as a major neurodevelopmental risk factor (Niwa et al, 2016) and use mutant DISC1, a C-terminustruncated form of full-length protein, as a dominant-negative molecular tool to elucidate the role of DISC1 role in astrocytes.

Our prior study demonstrates that astrocytic DISC1 binds to and stabilizes serine racemase (SR), the enzyme that converts L-serine to D-serine, a N-methyl-D-aspartate receptor (NMDAR) co-agonist (Ma et al, 2013). Selective expression of C-terminal-truncated human DISC1 (mutant DISC1) in astrocytes decreases levels of endogenous mouse 
DISC1 in a dominant-negative manner, resulting in disruption of DISC1-SR binding, increased SR ubiquitination, and decreased D-serine production by astrocytes. These biochemical changes are associated with an enhanced response to the non-competitive antagonist of NMDAR, MK-801, that was reversed by $\mathrm{D}$-serine treatment, suggesting a functional link between expression of mutant DISC1 in astrocytes, decreased D-serine production, and behavioral alterations (Ma et al, 2013).

In identifying the pathophysiological mechanisms of psychiatric dysfunction, research has shed light on a significant contribution of adult neurogenesis (Jun et al, 2012b; Schoenfeld and Cameron, 2015). Adult hippocampal neurogenesis describes the active process in which adultborn neurons are continuously generated from neural stem cells (NSCs) throughout life in the dentate gyrus (DG). Normal proliferation and maturation of local NSCs in the DG contributes to affective behaviors and cognitive function (Christian et al, 2014), and impairments in these processes are thought to underlie anxiety, mood disorders, and/or learning and memory in several neuropsychiatric disorders (Christian et al, 2014; Clelland et al, 2009; Snyder et al, 2011; Zhou et al, 2013; Revest et al, 2009).

Previous studies have implicated DISC1 in maintaining proper dendrite morphogenesis of newborn neurons during adult hippocampal neurogenesis (Duan et al, 2007) and neural differentiation of DG granule cells (Enomoto et al, 2009). Intriguingly, astrocytes are demonstrated to play a critical role in regulating neurogenesis by secreting $\mathrm{D}$-serine (Sultan et al, 2015). Thus, we hypothesized that expression of mutant DISC1 in astrocytes would decrease D-serine production in the hippocampus, leading to impaired adult hippocampal neurogenesis and associated behavioral abnormalities. We found that expression of mutant DISC1 in astrocytes increased anxiety, attenuated social interaction and preference for social novelty and impaired cognitive behaviors in mice. The behavioral phenotypes were associated with decreased proliferation of neural progenitors, and diminished dendrite outgrowth of dentate granule newborn neurons in mice. Consistent with observed decreased levels of $\mathrm{D}$-serine in the hippocampus of mutant DISC1 mice, treatment with D-serine ameliorated the altered behaviors and restored abnormal development of newborn neurons. Our findings demonstrate for the first time that DISC1 expressed in mature astrocytes is involved in regulation of adult hippocampal neurogenesis and hippocampusdependent affective and cognitive behaviors.

\section{MATERIALS AND METHODS}

\section{Tet-off Transgenic Model}

Our prior study used the GFAP-tTA line (Jackson Lab line 110, B6.Cg-Tg(GFAP-tTA)110Pop/J) that expresses mutant DISC1 at embryonic and early postnatal stages (Ma et al, 2013). Thus, the neurobehavioral alterations observed in that study were likely related to effects of mutant DISC1 on astrocytes that continue to develop during first weeks of postnatal development (Ge et al, 2012; Kanski et al, 2014). In order to assess the role of astrocytic DISC1 in adult hippocampal neurogenesis, we expressed mutant DISC1 in mature astrocytes using the GFAP-tTA line 67 (a kind gift by
Dr. Brian Popko, University of Chicago) that, in contrast to line 110 ( $\mathrm{Ma}$ et al, 2013), drives expression of mutant DISC1 predominantly during adolescence and adulthood (Supplementary Figure S1A).

GFAP-tTA mice were crossed with single-transgenic TREmutant DISC1 mice (line 1001) as previously described (Ma et al, 2013). All mice were on the C57BL/6 background. This mating protocol produces litters composed of $\sim 50 \%$ single-transgenic mice not expressing mutant DISC1 (control mice) and $\sim 50 \%$ double transgenic mice-expressing mutant DISC1 (mutant mice). Tail tissue samples were used for genotyping as previously described (Pletnikov et al, 2008). Developing mice were housed with their dams until postnatal days (P) 21-23 with food and water ad libitum. All procedures were approved by the JHU Animal Care and Use Committee.

\section{Behavioral Tests}

Behavioral tests were performed on control and mutant DISC1 mice starting at 2 months of age. The interval between different behavioral tests was at least 1 week. Different batches of mice were used for different tests to minimize over-training that could affect neurogenesis. Specifically, two non-stressful tests and one stressful test were combined for each batch of mice. Non-stressful tests include noveltyinduced activity in open field, elevated plus maze (EPM), spontaneous alternation and spatial recognition in a Y-maze, novel object recognition test (NORT), the 3-chamber test for social interaction and radial arm maze (RAM). Stressful tests included pre-pulse inhibition (PPI) of the acoustic startle response, forced swim test (FST), and trace fear conditioning. The methods used for open field activity, EPM, Y-maze, NORT, social interaction, and PPI have been described in detail in our previous publications (Abazyan et al, 2014a; Abazyan et al, 2010; Ayhan et al, 2011; Pletnikov et al, 2008).

To measure depression-like behaviors in FST, mice were placed in a cylinder filled halfway with room temperature water for $6 \mathrm{~min}$. The last $4 \mathrm{~min}$ of the test were scored for immobility time.

For RAM, mice were food deprived to reach $80 \%$ of original body weight. Mice were then habituated for five minutes to an eight arm-radial maze (Med Associates, Georgia, VT) with fruit loops at the end of each arm. During the 8-day training period, four arms contained fruit loops (baited arms) and four arms were empty (non-baited arms). Mice were given $5 \mathrm{~min}$ on each training day to find and eat the fruit loops in the four baited arms. Forty-eight hours following the last training day, mice were tested for retention test for working and reference memory. Working memory errors were defined as entries into a baited arm after the bait has already been acquired. Reference memory errors were defined as entries into non-baited arms.

Trace fear conditioning was a 3-day test consisting of a habituation day, training day, and a test day. Mice were habituated to the shock box (Coulbourn, Holliston, MA) for $10 \mathrm{~min}$. The following day, mice were placed in the shock box, and a 20-s white noise tone was delivered. Twenty seconds following the termination of the tone, a scrambled 2-s $0.5 \mathrm{~mA}$ shock was delivered. This tone-shock pairing was repeated three times. On the third day, mice were placed in the shock box for $3 \mathrm{~min}$ to measure freezing in response to the context. Following this, mice were placed in a separate 
context and the 20-s white noise tone was delivered, during which freezing in response to the tone was measured.

\section{Tissue Preparation, Immunostaining, Confocal Imaging, and Analysis}

To analyze neural progenitor proliferation, adult mice were anesthetized with a mixture of ketamine $(100 \mathrm{mg} / \mathrm{kg})$, xylazine $(20 \mathrm{mg} / \mathrm{kg})$, and acepromazine $(10 \mathrm{mg} / \mathrm{kg})$ and perfused with $4 \%$ paraformaldehyde (PFA). The harvested brain samples were postfixed with $4 \%$ PFA and equilibrated in $30 \%$ sucrose. Coronal brain sections were cut at $40 \mu \mathrm{m}$ using a sliding microtome (Leica SM 2010 R) and every fifth section collected and processed for immunostaining. For MCM2 immunostaining, an antigen retrieval procedure was performed as previously described (Jang et al, 2013). All sections were counterstained with 4', 6-diaminodino-2phenylindole (DAPI).

In order to determine cell type-specific activity of the GFAP promoter, we mated GFAP-tTA mice (line 67) with TRE-LacZ (JAX Stock No: 002621 B6;SJL-Tg(tetop-lacZ) $2 \mathrm{Mam} / \mathrm{J}$ ) or TRE-tdTomato (a gift of Dr. Hongkui Zeng, the Allen Institute for Brain Science, Seattle, WA) mice. Brain sections from double transgenic mice were co-stained with the following antibodies: GFAP, S100 $\beta$, NeuN, $\beta$-galactosidase (LacZ), or tdTomato antibodies (Supplementary Table 1). Images were acquired on a Zeiss LSM 780 confocal system (Carl Zeiss) with $\times 20, \times 40$ objective lens using a multitrack (z-stack and tile scanning) configuration.

For neural progenitor proliferation analysis, an antigen retrieval protocol was performed using a microwave as previously described (Hussaini et al, 2013; Jang et al, 2013). Briefly, citrate buffer (1.8 mM citric acid, $8.2 \mathrm{mM}$ tri-sodium citrate) was pre-heated for $5 \mathrm{~min}$ at maximum power. Brain sections were then placed in hot citrate buffer and incubated for another $7 \mathrm{~min}$ at maximum power. Brain sections were immunostained with MCM2 antibody. Stereological quantification of $\mathrm{MCM}^{+}$cells within the subgranular zone (SGZ) were carried out as previously described (Jang et al, 2013).

\section{Stereotaxic Injection of Engineered Retroviruses}

Engineered self-inactivating murine onco-retroviruses were used to express GFP specifically in proliferating cells and their progeny (Duan et al, 2007; Jang et al, 2013). High titers of engineered retroviruses were produced by co-transfection of retroviral vectors and VSVG into GP2-293 cells followed by ultra-centrifugation of viral supernatant. Once anaesthetized, retroviruses were stereotaxically injected into the DG at four sites $(0.5 \mu \mathrm{l}$ per site at $0.1 \mu \mathrm{l} / \mathrm{min})$ with the following coordinates (in $\mathrm{mm}$ from bregma): anterioposterior $=-2$; mediolateral $= \pm 1.6$; dorsoventral $=2.3$ and anterioposterior $=-3$; mediolateral $= \pm 2.6$; dorsoventral $=3.3$ as previously described with slight modification (Duan et al, 2007; Ge et al, 2006; Jang et al, 2013). Mice were sacrificed at 14 or 28 day post-injection for morphological analysis as previously stated (Jang et al, 2013). Briefly, all sections were immunostained with chicken-GFP and three-dimensional reconstructions of the dendritic processes of each $\mathrm{GFP}^{+}$ neuron were made from $\mathrm{Z}$-series stacks of confocal images. The projection images were semi-automatically traced with $\mathrm{NIH}$ ImageJ using the NeuronJ plugin. The total dendritic length and branch number of individual $\mathrm{GFP}^{+}$neurons were subsequently analyzed. Averages of total dendritic length of each individual neuron under different conditions was measured (Duan et al, 2007; Ge et al, 2006; Jang et al, 2013).

\section{Doxycycline Treatment and Dendritic Maturation}

GFAP-tTA mice and TRE-mutant DISC1 mice (line 1001) were crossed, pregnant female mice and their offspring (both control and mutant DISC1 mice) were maintained on Doxycycline (DOX) food (Bio-Serv, Frenchtown, NJ, $200 \mathrm{mg} / \mathrm{kg}$ ) until 6 weeks as previously described (Ayhan et al, 2011). At 6 weeks of age, control and mutant DISC1 mice were injected with engineered self-inactivating murine onco-retroviruses as above and immediately after the surgery were placed on regular food. After 2 weeks, mice were killed and the total dendritic length of individual $\mathrm{GFP}^{+}$neurons was analyzed as above.

\section{Western Blotting}

Expression of mutant DISC1 was measured in forebrain area of the embryonic brain (E18), newborn mouse brain (P1), or hippocampi dissected form the mouse brain at P14 or 56 with anti-myc antibody as previously described (Pletnikov et al, 2008). Expression of SR was assayed at P30 with custom-made antibody as previously described (Ma et al, 2013). The optical density (O.D.) of protein bands on each digitized image was normalized to the O.D. of the loading control ( $\beta$-actin, 1:20000, Sigma-Aldrich, MO). Densitometry was done using ImageJ software. Normalized values were used for analyses.

\section{D-serine Assay and Treatments}

D-serine was measured in frontal parts of the brain at P0-3 and hippocampal samples at P30 in control and mutant DISC1 mice using HPLC as previously described (Grant et al, 2006; Ma et al, 2013).

D-serine was administered using published protocols (Balu et al, 2013; Sultan et al, 2013) with slight modifications. Specifically, control and mutant DISC1 mice were given once daily, subcutaneous injections of vehicle or $\mathrm{D}$-serine for 39 days. D-serine was administered at a dose of $300 \mathrm{mg} / \mathrm{kg}$ on day 1 followed by $150 \mathrm{mg} / \mathrm{kg}$ for the remaining days. The day after cessation of $\mathrm{D}$-serine treatment, potential ameliorative effects of D-serine were assessed in the EPM test, FST, and trace fear conditioning test. Two cohorts of mice were used, with one being tested in the EPM test and the FST, and the other one being evaluated in the trace fear conditioning test. Mice were killed after the EPM and FST to evaluate the effects of D-serine treatment on dendrite development of newborn neurons in the DG.

\section{Statistical Analyses}

Results are expressed as the mean \pm standard error of the mean (SEM). The behavioral results were analyzed using analyses of variance (ANOVA) or Student $t$-test when appropriate. The effects of mutant DISC1 on proliferation, neuronal differentiation, or dendrite development were analyzed with two-tailed Student $t$-test or Wilcoxon non- 
parametric test, if the normal distribution test failed. $p<0.05$ was used for the significance level.

\section{RESULTS}

\section{Astrocytic Mutant DISC1 Induces Abnormal Anxiety- Like and Depression-Like Behavior}

Our prior study demonstrated that late gestation and perinatal expression of mutant DISC1 in astrocytes produced enhanced responses to the psychostimulant, MK-801, without altering anxiety-, or depression-related behaviors (Ma et al, 2013). The present study uses the GFAP-tTA promoter line (line 67) that is active during adolescence and adulthood in mature astrocytes (Supplementary Figure S1). As our pilot tests indicated increased anxiety-like behavior in EPM and depression-like behavior in FST in female but not male mutant mice (data not shown), this study focused on female mutant DISC1 mice.

Mutant DISC1 mice exhibited decreased time in the open arms of the EPM, indicating elevated anxiety-like behavior (Figure 1a), and increased immobility in the FST indicating a potential increase in depression-like behavior (Figure 1b), and attenuated sociability and social novelty preference (Figure 1c and Supplementary Figure S2). We observed no significant group differences in novelty-induced locomotor activity in open field (Supplementary Figure S3A) or PPI of the acoustic startle response (Supplementary Figure S3B). Mutant DISC1 did not affect spontaneous alternations or spatial recognition memory in Y-maze (Supplementary Figure S3C-D), novel object recognition (Supplementary Figure S3E) or context- or cue-dependent fear conditioning (Supplementary Figure S4).

As we saw no effects of mutant DISC1 on learning and memory in simple tasks, we decided to use more complex cognitive tests that have been found to place a greater demand on the proper functioning of the hippocampus (Laroche et al, 2000). In contrast to the simple version of fear conditioning, in the trace fear conditioning task, mutant DISC1 mice exhibited decreased freezing behavior in response to a cue compared to control animals (Figure 1d), with no group effects noted on the freezing behaviors during the training session or the context-dependent conditioning (Supplementary Figure S5A-B). Similarly, in RAM, another complex hippocampus-dependent cognitive task, mutant DISC1 mice displayed increased latency to visit the correct arm during the probe trial (Figure 1e) and made significantly more working memory and reference memory errors (Supplementary Figure S5C-D), with the performance during the training session being comparable between two groups (Supplementary Figure S5E). Thus, our behavioral tests indicate that expression of mutant DISC1 in astrocytes affects both emotion-related and cognitive behaviors in mice.

\section{Astrocytic Mutant DISC1 Suppresses Proliferation of Neural Progenitors in the Adult DG}

As the hippocampus is one brain region with high expression of mutant DISC1 in astrocytes (Ma et al, 2013), we sought to explore the effects of mutant DISC1 on adult hippocampal neurogenesis, where potential abnormalities could contribute to the observed abnormalities in affective and cognitive behaviors in mutant DISC1 mice. In the adult mouse DG, neural progenitors reside and proliferate within the SGZ that subsequently differentiate into mature dentate granule neurons (Christian et al, 2014; Kempermann et al, 2004). To examine the potential role of astrocytic mutant DISC1 in proliferation of neural progenitors, we performed immunostaining with mini-chromosome maintenance complex component 2 (MCM2) as a marker for endogenous cell proliferation (Jang et al, 2013). We found a significantly decreased number of $\mathrm{MCM}^{+}$cells in the SGZ of the mutant
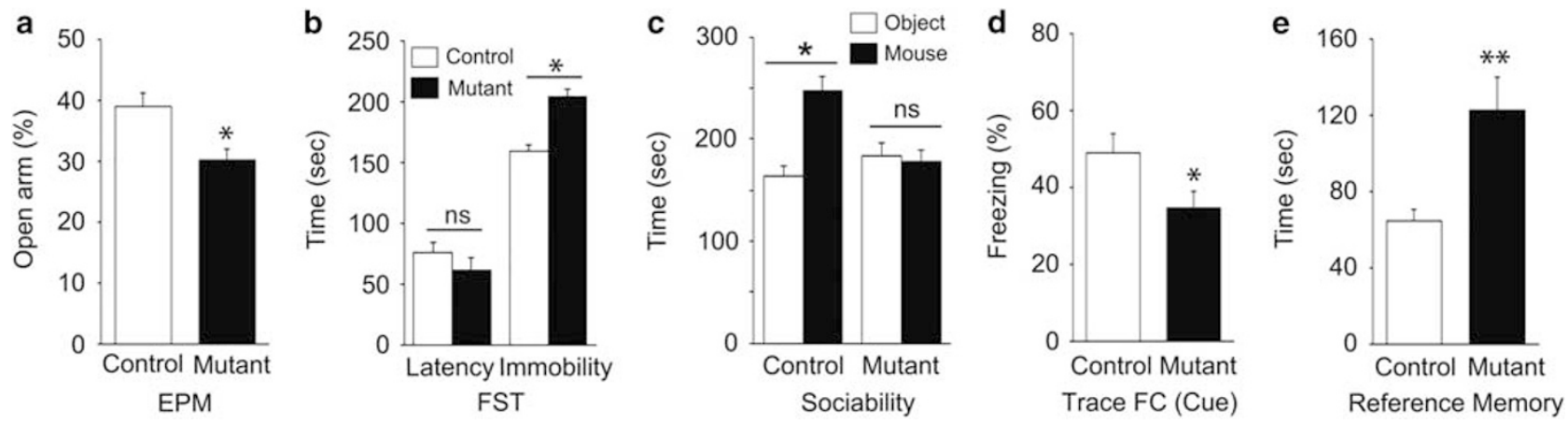

Figure I Expression of mutant DISCI in astrocytes elevates anxiety, attenuates social behaviors, and impairs cognitive function. (a) Compared to control littermates, mutant DISCI mice spent significantly less time in the open arms of the elevated plus maze (EPM). Values represent mean \pm SEM $(n=16-23$ mice/ group; ${ }^{*} p=0.005$, control vs mutant mice; Student two-tailed $t$-test, $\left.t=2.997\right)$. (b) Compared to control littermates, mutant DISCI mice demonstrated significantly increased immobility time in the forced swim test (FST). Values represent mean \pm SEM $(n=16-23$ mice/group; * $p=0.006$, control vs mutant mice, Student two-tailed t-test, $t=3.079$; ns - no group-dependent significant difference in the latency to the first immobility episode. (c) Compared to control littermates, mutant DISCI mice exhibited decreased sociability. Values represent mean \pm SEM, $n=10-13$ mice; * $p<0.05$ for inanimate object vs mouse object in control mice; two-way repeated measures ANOVA showed a significant group effect, $F(I, 45)=6.67, p=0.017$, significant effect of subject-object time, $F(I, 45)=7.24, p=0.014$, and the group by subject-object time interaction, $F(I, 45)=9.76, p=0.005$. Post hoc comparisons (Bonferroni $t$-test) showed a significant difference in time spent with the live mouse vs inanimate object for control mice $(p<0.00 \mathrm{I})$ but not mutant mice, $p=0.78$. ns - no significant difference in exploring the object vs live mouse in mutant DISCI mice. (d) Compared to control littermates, mutant DISCI mice showed a significantly reduced cue-dependent freezing behavior in trace fear conditioning. Values represent mean \pm SEM ( $n=10$ mice/group; * $p=0.042$, control vs mutant mice, Student two-tailed $t$-test, $t=8.1 \mathrm{I}$ ). (e) Compared to control littermates, mutant DISCI mice had significantly longer latency to find the correct arm in RAM. Values represent mean \pm SEM $(n=10-17$ mice/group; $* * * 0.01$, control vs mutant mice, Student two-tailed $t$-test, $t=10.9)$. 
DISC1 mice (Figure 2a and b), suggesting that expression of mutant DISC1 in astrocytes suppresses proliferation of neural progenitors in the adult DG.

\section{Astrocytic Mutant DISC1 Inhibits Dendrite Development of Newborn Dentate Granule Neurons}

During the maturation process, newborn neurons rapidly extend dendrites into the molecular layer to form synapses and incorporate into existing hippocampal circuits contributing to hippocampal function (Kempermann et al, 2004). To evaluate the effects of astrocytic mutant DISC1 on dendrite development, we analyzed dendrite morphological changes using a GFP-expressing retrovirus-mediated approach for birth-dating proliferating cells and their progeny (Ge et al, 2006; van Praag et al, 2002) (Figure 3a). We found a significant decrease in the total dendrite length of $\mathrm{GFP}^{+}$ newborn neurons in mutant DISC1 mice compared to control animals at 4 weeks post-injection (wpi) (Figure 3b and c), suggesting that mutant DISC1 impairs dendrite development in the adult hippocampal DG.

Since GFAP is also expressed by NSCs in the adult hippocampus (Kempermann et al, 2004), we evaluated possible expression of mutant DISC1 in NSCs. In order to assess activity of the GFAP-tTA line in different cell types in the DG, we used TRE reporter lined that expresses $\beta$-galactosidase (LacZ) or tdTomato and found co-localiz-

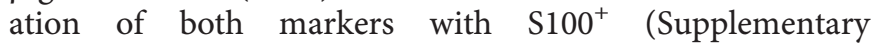
Figure S6A) and $\mathrm{GFAP}^{+}$cells (Supplementary Figure S6B). Stereological analysis revealed the majority of $\mathrm{LacZ}^{+}$cells were co-labeled with mature astrocytes defined by $\mathrm{GFAP}^{+}$ cells with star-shape morphology in the hippocampal area ( $~ 99.69 \pm 0.34 \%$ in the hilus, and $\sim 94.32 \pm 1.57 \%$ in the DG), suggesting that mutant DISC1 co-expressed with mature astrocytes. We also observed that a small portion of mutant DISC1 co-expressed with NSCs defined by $\mathrm{LacZ}^{+} \mathrm{GFAP}^{+}$ cells with radial glial-like (RGL) morphology within the SGZ $(\sim 7.97 \pm 1.53 \%$, Supplementary Figure S6C), indicating that the overwhelming majority of mutant DISC1 expressing cells are hippocampal mature astrocytes. Notably, no LacZ co-labeling with neuronal marker NeuN was observed (0\%; mature neurons, Supplementary Figure S6D), indicating a cell type-specific expression of mutant DISC1 in our mouse system. Supplementary Figure S7 provides a summary of these results.

In order to rule out the possibility that even a small portion of NSCs expressing mutant DISC1 could contribute to the observed abnormalities in adult neurogenesis, we manipulated expression of mutant DISC1 with regard to time when newborn cells are no longer GFAP expressing NSCs and have become post-mitotic neurons (Goncalves et al, 2016; Kang et al, 2016; Sultan et al, 2015). Specifically, we bred and kept control and mutant DISC1 mice on DOX diet until 6 weeks of age. We then injected GFP-expressing retrovirus in the hippocampus as above and replaced the DOX diet with a regular one immediately after the surgery. In this way, expression of mutant DISC1 was restored in astrocytes 5 days after virus injection (Supplementary Figure S8). We analyzed dendritic development of newborn $\mathrm{GFP}^{+}$neurons in these mice at 2 wpi (Figure $3 e$ and f). Similar to the results reported above, we found a significant reduction in the total dendritic length of $\mathrm{GFP}^{+}$newborn neurons in mutant
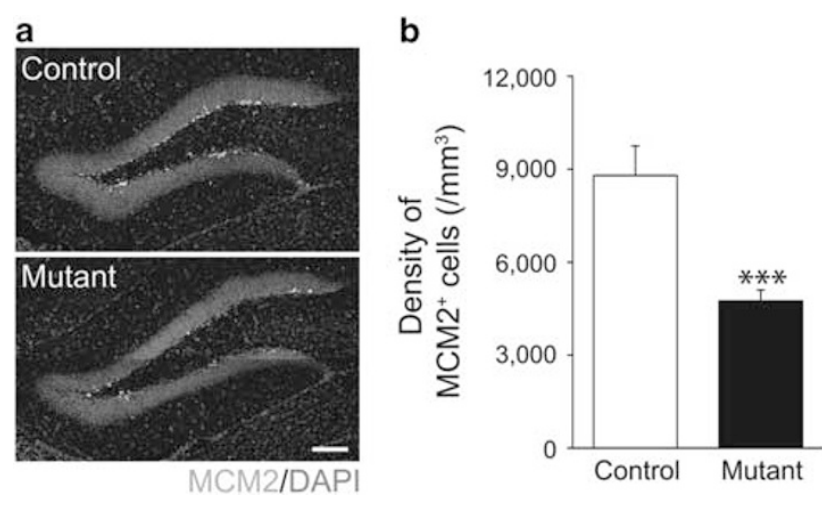

Figure 2 Expression of mutant DISCI in astrocytes reduces proliferation of neural progenitors. (a) Representative confocal image of MCM2 immunostaining (endogenous cell proliferation marker; green) and DAPI (red) in the dentate gyrus (DG) of adult control and DISCI mutant mice. Scale bar: $100 \mu \mathrm{m}$. (b) Stereological quantification of $\mathrm{MCM}^{+}$cells in the adult subgranular zone (SGZ). Value represents mean \pm SEM $(n=7$ control and $n=8$ mutant DISCI mice; **** $p<0.001$; Student two-tailed t-test, $t=4.567)$. A full color version of this figure is available at the Neuropsychopharmacology journal online.

DISC1 mice compared to control animals. Our data suggest that observed reduction in dendrite growth was unlikely due to scarce expression of mutant DISC1 in NSCs of the DG.

\section{D-serine Ameliorates Affective Behaviors in Mutant DISC1 Mice}

Our prior study demonstrates decreased tissue levels of Dserine in mice that express mutant DISC1 in astrocytes. Consistent with the in vitro report (Abazyan et al, 2014b) we found no significant effects of mutant DISC1 on the numerical density of astrocytes in the hilus of the DG (Supplementary Figure S9), suggesting that lower levels of Dserine do not result from decreased numbers of astrocytes in the hippocampus of adult mice.

When we used a different GFAP-tTA line (line 110) that drives expression of mutant DISC1 predominantly in developing mice, we observed decreased levels of D-serine in newborn mutant mice but detected no significant changes in D-serine levels at P30 when the activity of the GFAP promoter (line 110) is low (Ma et al, 2013). As the present study employs the GFAP-tTA line (line 67) that drives expression of mutant DISC1 in adult mice, we first decided to confirm the effects of mutant DISC1 on SR expression and D-serine levels in the hippocampus in mutant mice at P30. We found a significant decrease in SR expression (Supplementary Figure S10A) and D-serine level (Supplementary Figure S10B) in mutant DISC1 mice compared to control animals. Notably, consistent with low neonatal expression of mutant DISC1 in line 67 (Supplementary Figure S1), no significant changes in $\mathrm{D}$-serine levels were observed in newborn mutant DISC1 mice generated by crossing GFAPtTA line 67 with TRE-mutant DISC1 mice (Supplementary Figure S10C). Taken together, our published and present results indicate that astrocytic mutant DISC1 diminishes D-serine production in a dose-dependent manner.

We hypothesized that abnormal postnatal production of D-serine in the hippocampus could impair adult hippocampal 
a

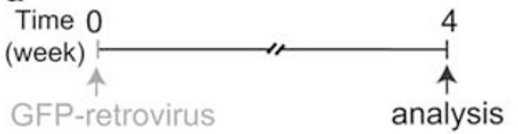

b

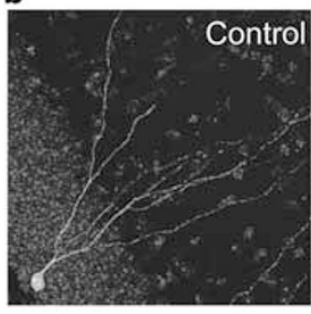

d

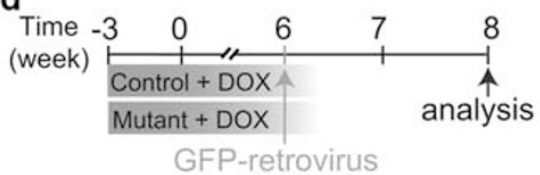

e

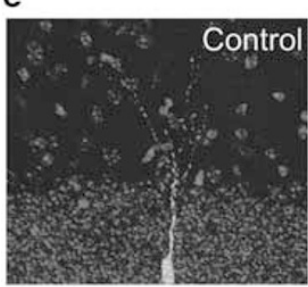

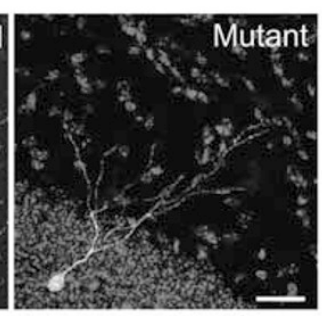

GFP-retrovirus/DAPI analysis

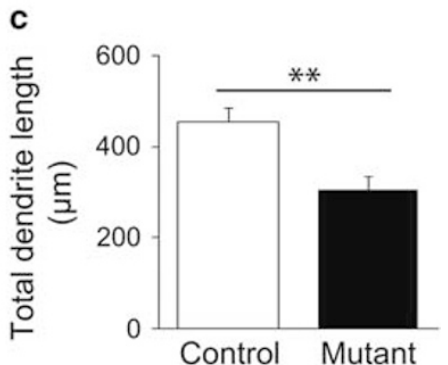



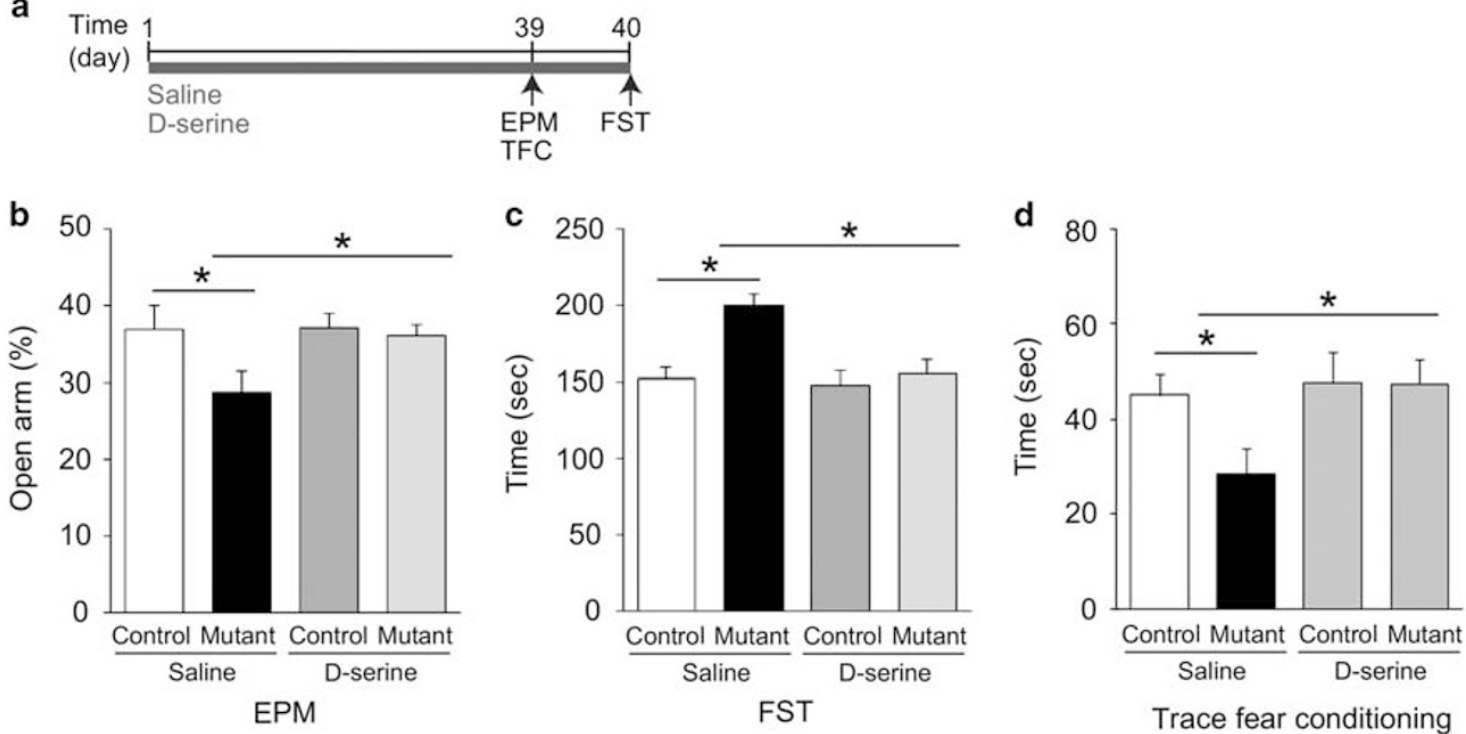

Figure 4 D-serine treatment ameliorates the behavioral abnormalities in mutant DISCI mice. (a) A schematic diagram of the experimental design. 8 weekold mice were injected with D-serine as described in the Methods. One cohort of mice was tested in the elevated plus maze (EPM) and forced swim test (FST); a separate cohort of mice was tested in trace fear conditioning. (b) D-serine treatment decreased anxiety-like behavior in DISCI mutant mice in EPM. Values represent mean \pm SEM ( $n=5-7$ mice per group); two-way ANOVA revealed no significant interaction $(F(I, 2 I)=1.53, p>0.05)$. Planned t-tests indicated a significant difference between saline-treated control and mutant DISCI mice, $p<0.05$; and a significant difference between D-serine- treated and saline-treated mutant mice, $t=2.26, p<0.05$; $* 2<0.05$. (c) D-serine treatment decreased immobility in DISCI mutant mice in FST. Values represent mean \pm SEM $(n=6-7$ mice per group); two-way ANOVA showed a significant effect of genotype, $F(I, 24)=9.8, p=0.005$, a significant treatment effect, $F(I, 24)=7.5, p=0.012$, and the genotype by treatment interaction, $F(I, 24)=5.2, p=0.034$. Post hoc Fisher LSD test showed a significant difference between saline-treated and D-serinetreated mutant DISCI mice $(p<0.05)$, and a significant difference between saline-treated control and mutant DISCI mice $(p<0.05)$. (d) D-serine treatment improved performance of mutant DISCI mice in the trace fear conditioning test as evidenced by the increased time of the cue-dependent freezing behavior. Values represent mean \pm SEM ( $n=6-7$ mice per group); two-way ANOVA showed a significant effect of genotype, $F(1,24)=4.36, p=0.04$ and the genotype by treatment interaction, $F(I, 24)=5.34, p=0.028$. Post hoc Fisher LSD showed significant differences between saline-treated control and saline-treated mutant DISCI mice $(p<0.05)$, and a significant differences between D-serine- treated and saline-treated mutant DISCI mice $(p<0.05)$, $*<<0.05$.

2015) and are in line with prior studies that have implicated abnormalities in adult neurogenesis in several neuropsychiatric disorders (Kempermann and Kronenberg, 2003). For example, inhibition of adult neurogenesis abolishes the behavioral effects of antidepressants (Santarelli et al, 2003), causes fear related behaviors (Kheirbek et al, 2012), and affects learning and memory (Cameron and Glover, 2015; Gould et al, 1999). However, there are also a number of negative reports on the effects of adult neurogenesis on affective behaviors and/or responses to antidepressants (Fuss et al, 2010; Jun et al, 2012a; Wang et al, 2008). It is, nevertheless, conceivable that one of the possible mechanisms whereby dysfunctional astrocytes contribute to cognitive impairment and mood disorders is through affecting adult neurogenesis.

Astrocytes are key niche players controlling NSC development in the adult hippocampus, including NSC proliferation and differentiation towards neurogenesis (Ma et al, 2005; Song et al, 2002). However, the exact molecular mechanisms by which astrocytes regulate adult neurogenesis are incompletely understood, although the majority of relevant studies have indicated that secreted factors seem to mediate the influence of astrocytes on adult neurogenesis (Clarke and Barres, 2013; Hamilton and Attwell, 2010; Parpura et al, 2012; Song et al, 2002). One of the soluble factors secreted by astrocytes is D-serine, a co-agonist of NMDAR (Radzishevsky et al, 2013). Although the initial reports suggested astrocyte localization for SR (Wolosker et al, 1999), the recent studies using Srr genetic models have demonstrated strong expression of SR in neurons (Wolosker et al, 2016). However, deleting SR in forebrain neurons leads to only $50 \%$ decrease in extracellular levels of D-serine, suggesting a non-neuronal contribution (Ishiwata et al, 2015). In addition, the online RNA-Seq transcriptome database indicates that compared to neurons, non-neuronal brain cells express mRNA for Srr at higher levels (Zhang et al, 2014). D-serine increases the proliferation of NSC in vitro and in vivo (Sultan et al, 2013). Expression of mutant DISC1 in astrocytes results in decreased production of Dserine, possibly through increased ubiquitination of SR (Ma et al, 2013). As treatment with D-serine rescues abnormal neurogenesis and differentiation of newborn neurons and ameliorates the behavioral abnormalities in mutant DISC1 mice, it is tempting to speculate that abnormalities in Dserine metabolism in the hippocampus of mutant DISC1 mice might be at least in part responsible for the decreased adult hippocampal neurogenesis and associated abnormal behaviors.

In contrast to previous reports, our study is the first to provide experimental evidence for the role of astrocytic DISC1 in adult neurogenesis. Prior study reports retroviral shRNA knockdown of mouse DISC1 in neural progenitors promotes dendritic development in vivo (Duan et al, 2007). In addition to the different target (neurons $v s$ astrocytes), the shRNA treatment has been suggested to acutely affect the development of newborn neurons that may explain the 
a

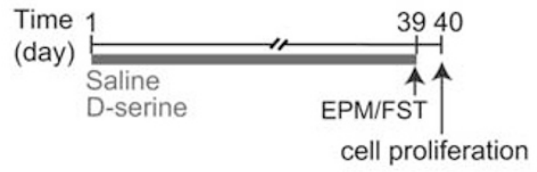

b
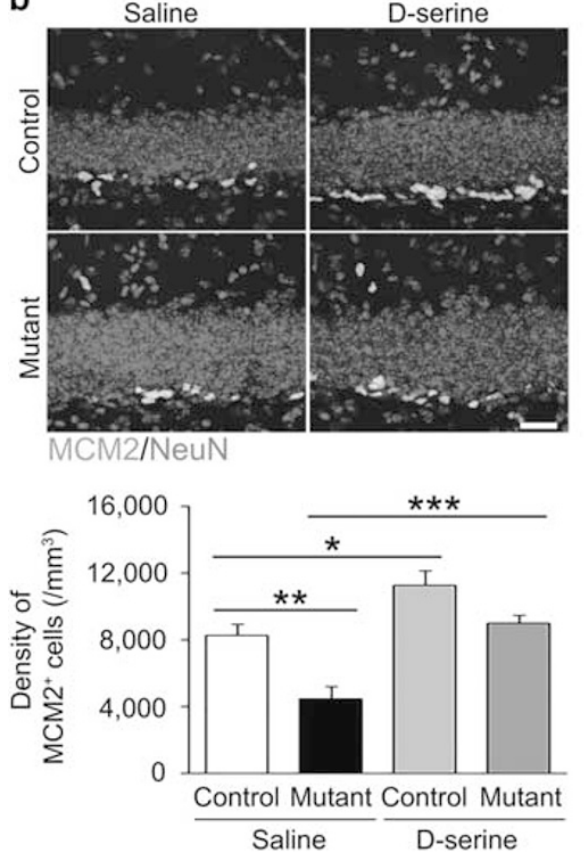

C
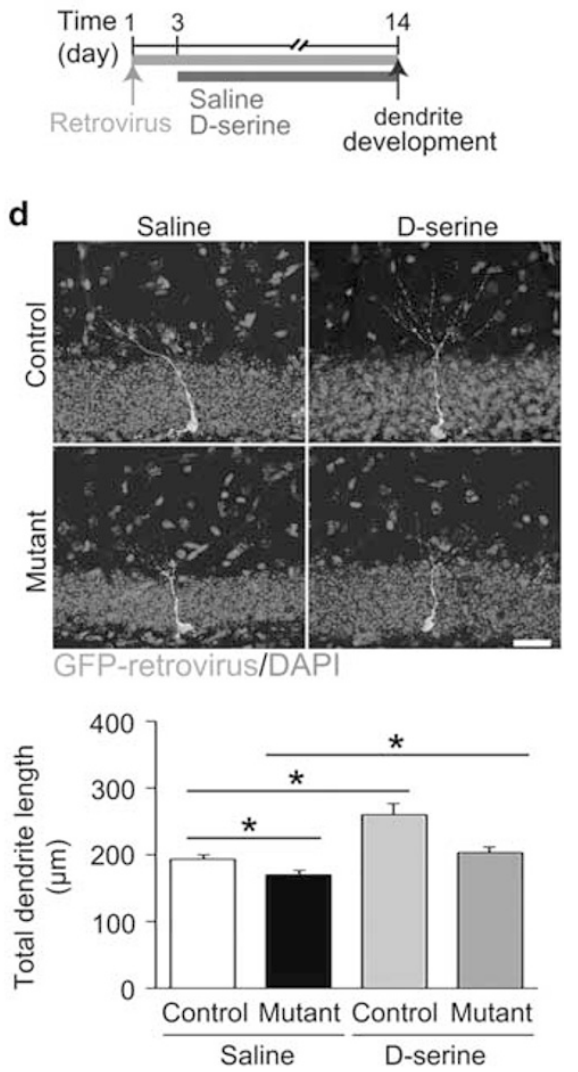

Figure 5 D-serine treatment restores reduced progenitors proliferation and dendrite growth of newborn neurons in mutant DISCI mice. (a,b) D-serine treatment increases $\mathrm{MCM2}^{+}$neural progenitor proliferation in mutant DISCI mice. (a) A schematic diagram of the experimental design for proliferation analysis. (b) Top: representative confocal images of MCM2 (green) and DAPI (red) staining. Scale bar: 20 um. Bottom: a summary of stereological quantification of proliferating $\mathrm{MCM}^{+}$neural progenitors. Values represent mean \pm SEM ( $n=5$ mice for control treated with saline group, $n=7$ for mutant treated with saline, $n=6$ mice for control treated with $D$-serine group, and $n=7$ for mutant treated with D-serine group). Two-way ANOVA showed a significant effect of D-serine treatment, $F(I, 22)=44.5, p<0.00 I$, and the significant group by treatment interaction, $F(I, 22)=6.1, p=0.023$; post hoc Fisher LSD test showed a significant difference between saline-treated control and mutant DISCI mice $(p<0.0 \mathrm{I})$, a significant difference between saline-treated and D-serine-treated mutant DISCI mice $(p<0.00 \mathrm{I})$ as well as a significant difference between saline-treated and D-serine-treated control mice $(p<0.05)$; ${ }^{*} p<0.05$, ** $p<0.0 \mathrm{I}$; ***** $p<0.00$ I. (c,d) D-serine treatment restores dendritic growth of newborn dentate granule cells in mutant DISCI mice. (c) A schematic diagram of the experimental design for analyzing dendrite development. (d) Top: Representative confocal images of GFP newborn neurons (green) and DAPI staining (red). $\mathrm{GFP}^{+}$neurons were examined at 2 weeks post-injection in order to avoid ceiling effects of dendrite growth by D-serine treatment. Scale bar: 20 um. Bottom: quantification of the total dendritic length of $\mathrm{GFP}^{+}$dentate granule cells. Values represent mean $\pm \mathrm{SEM}(n=63$ neurons from three control saline-treated mice; $n=83$ neurons from five mutant DISCI saline-treated mice; $n=133$ neurons from nine control D-serine-treated mice; and $n=184$ neurons from nine mutant DISCI D-serine-treated mice); two-way ANOVA showed a significant effect of genotype, $F(I, 26)=27.9, p<0.00 I$, and a significant effect of D-serine treatment, $F(I, 26)=50.76, p<0.00 I$, with no significant interaction. Planned $t$-tests showed a significant difference between saline-treated control and mutant DISCI mice $(p<0.05)$, a significant difference between saline-treated and D-serine-treated mutant DISCI mice $(p<0.05)$ as well as a significant difference between saline-treated and D-serine-treated control mice $(p<0.05)$; $p<0.05$. A full color version of this figure is available at the Neuropsychopharmacology journal online.

differences between germ-line and viral vector models. Indeed, several germ-line DISC1 animal models that evaluated the role of DISC 1 in adult neurogenesis reported the changes consistent with the present results. Altered positioning and numbers of immature neurons and cytoarchitecture alterations in mature granule cells as well as changes in the generation, placement, and maturation of newly generated neurons in the hippocampal DG have been reported for those mouse models (Chandran et al, 2014; Kvajo et al, 2008). Our study suggests a new mechanism whereby DISC1 variants within astrocytes can affect neurogenesis through decreasing secretion of D-serine.

Our study also points to a possible reason for the heterogeneity of diagnostic categories associated with DISC1 variants. Indeed, despite its name, affective disorders were found in the original Scottish family and among the cases from other association studies (Blackwood et al, 2001; Blackwood et al, 2007; Millar et al, 2000b). It is conceivable that cell type-specific functions of DISC1 may determine varying clinical outcomes when pathogenic variants are expressed in neurons or glial cells.

Although only about $8 \%$ of $\mathrm{GFAP}^{+}$RGL NSCs express mutant DISC1, mutant DISC1 may have still affected NSC development. In order to directly test this possibility, we induced expression of mutant DISC1 in progenitor cells after they cease to be GFAP ${ }^{+}$and found that expression of mutant DISC1 continued to decrease dendritic development of newborn neurons in the DG. Thus, the effects of mutant DISC1 on adult neurogenesis and behaviors may not solely 
result from scarce expression of mutant DISC1 in NSCs of the SGZ.

Our results do not demonstrate a causal relationship between expression of mutant DISC1 in hippocampal astrocytes and abnormal adult neurogenesis and/or altered behaviors. We cannot rule out putative influence of extrahippocampal expression of mutant DISC1 on the behaviors. Although distant effects of factors secreted by astrocytes are possible, we think that the effects of mutant DISC1 on neurogenesis are likely exerted by hippocampal astrocytes rather than astrocytes located elsewhere in the brain as astrocytes predominantly regulate functions of neurons with which they make contact (Clarke and Barres, 2013; Molofsky et al, 2012). In a similar vein, the behavioral effects of $\mathrm{D}$ serine may have been also mediated by effects of $\mathrm{D}$-serine on neuronal populations elsewhere the brain. Future studies will address this possibility with intrahippocampal injections.

In conclusion, our results demonstrate that expression of mutant DISC1 in astrocytes increases affective behaviors and impairs adult neurogenesis and dendrite development of newborn neurons in the hippocampus. These behavioral and neuronal abnormalities are ameliorated with chronic treatment with D-serine. The findings suggest that genetic variants expressed in glial cells can contribute to emotional and cognitive dysfunction via affecting adult neurogenesis in the hippocampus.

\section{FUNDING AND DISCLOSURE}

This work was supported by MH-083728, MH-094268 The Conte Center grant, and The Brain and Behavior Research Foundation (former NARSAD) (MVP), Whitehall foundation, NARSAD, and Center for Regenerative Medicine at Mayo Clinic (MHJ), 5T32MH015330-38 Postdoctoral Fellowship Training Grant (CET; BA), Postdoctoral fellowship award from Center for Regenerative Medicine at Mayo Clinic (KHY), DA000266 (SHS). The authors declare no conflict of interest.

\section{ACKNOWLEDGMENTS}

We would like to thank Dr. Chan Il Choi for initial help with generating retrovirus, Dr. Sofya Abazyan for help with analysis of expression of mutant DISC1, Mrs. Olga Mychko for help with immunohistochemistry, and Mrs. Chunxia Yang with expert help with genotyping.

\section{REFERENCES}

Abazyan B, Dziedzic J, Hua K, Abazyan S, Yang C, Mori S et al (2014a). Chronic exposure of mutant DISC1 mice to lead produces sex-dependent abnormalities consistent with schizophrenia and related mental disorders: a gene-environment interaction study. Schizophr Bull 40: 575-584.

Abazyan B, Nomura J, Kannan G, Ishizuka K, Tamashiro KL, Nucifora F et al (2010). Prenatal interaction of mutant DISC1 and immune activation produces adult psychopathology. Biol Psychiatry 68: 1172-1181.

Abazyan S, Yang EJ, Abazyan B, Xia M, Yang C, Rojas C et al (2014b). Mutant disrupted-in-schizophrenia 1 in astrocytes: focus on glutamate metabolism. J Neurosci Res 92: 1659-1668.
Ayhan Y, Abazyan B, Nomura J, Kim R, Ladenheim B, Krasnova IN et al (2011). Differential effects of prenatal and postnatal expressions of mutant human DISC1 on neurobehavioral phenotypes in transgenic mice: evidence for neurodevelopmental origin of major psychiatric disorders. Mol Psychiatry 16: 293-306.

Balu DT, Li Y, Puhl MD, Benneyworth MA, Basu AC, Takagi S et al (2013). Multiple risk pathways for schizophrenia converge in serine racemase knockout mice, a mouse model of NMDA receptor hypofunction. Proc Natl Acad Sci USA 110: E2400-E2409.

Bernstein HG, Steiner J, Bogerts B (2009). Glial cells in schizophrenia: pathophysiological significance and possible consequences for therapy. Expert Rev Neurother 9: 1059-1071.

Blackwood DH, Fordyce A, Walker MT St, Clair DM, Porteous DJ, Muir WJ (2001). Schizophrenia and affective disorders-cosegregation with a translocation at chromosome 1q42 that directly disrupts brain-expressed genes: clinical and P300 findings in a family. Am J Hum Genet 69: 428-433.

Blackwood DH, Pickard BJ, Thomson PA, Evans KL, Porteous DJ, Muir WJ (2007). Are some genetic risk factors common to schizophrenia, bipolar disorder and depression? Evidence from DISC1, GRIK4 and NRG1. Neurotox Res 11: 73-83.

Cameron HA, Glover LR (2015). Adult neurogenesis: beyond learning and memory. Annu Rev Psychol 66: 53-81.

Chandran JS, Kazanis I, Clapcote SJ, Ogawa F, Millar JK, Porteous DJ et al (2014). Disc1 variation leads to specific alterations in adult neurogenesis. PLoS ONE 9: e108088.

Christian KM, Song H, Ming GL (2014). Functions and dysfunctions of adult hippocampal neurogenesis. Annu Rev Neurosci 37: 243-262.

Clarke LE, Barres BA (2013). Emerging roles of astrocytes in neural circuit development. Nat Rev Neurosci 14: 311-321.

Clelland CD, Choi M, Romberg C, Clemenson GD Jr., Fragniere A, Tyers P et al (2009). A functional role for adult hippocampal neurogenesis in spatial pattern separation. Science 325: 210-213.

Cotter DR, Pariante CM, Everall IP (2001). Glial cell abnormalities in major psychiatric disorders: the evidence and implications. Brain Res Bull 55: 585-595.

Duan X, Chang JH, Ge S, Faulkner RL, Kim JY, Kitabatake Y et al (2007). Disrupted-In-Schizophrenia 1 regulates integration of newly generated neurons in the adult brain. Cell 130: 1146-1158.

Enomoto A, Asai N, Namba T, Wang Y, Kato T, Tanaka M et al (2009). Roles of disrupted-in-schizophrenia 1-interacting protein girdin in postnatal development of the dentate gyrus. Neuron 63: 774-787.

Farrell MS, Werge T, Sklar P, Owen MJ, Ophoff RA, O'Donovan MC et al (2015). Evaluating historical candidate genes for schizophrenia. Mol Psychiatry 20: 555-562.

Fuss J, Ben Abdallah NMB, Hensley FW, Weber K-J, Hellweg R, Gass P (2010). Deletion of running-induced hippocampal neurogenesis by irradiation prevents development of an anxious phenotype in mice. PLoS ONE 5: e12769.

Ge S, Goh EL, Sailor KA, Kitabatake Y, Ming GL, Song H (2006). GABA regulates synaptic integration of newly generated neurons in the adult brain. Nature 439: 589-593.

Ge WP, Miyawaki A, Gage FH, Jan YN, Jan LY (2012). Local generation of glia is a major astrocyte source in postnatal cortex. Nature 484: 376-380.

Geschwind DH, Flint J (2015). Genetics and genomics of psychiatric disease. Science 349: 1489-1494.

Goncalves JT, Schafer ST, Gage FH (2016). Adult neurogenesis in the hippocampus: from stem cells to behavior. Cell 167: 897-914.

Gould E, Tanapat P, Hastings NB, Shors TJ (1999). Neurogenesis in adulthood: a possible role in learning. Trends Cogn Sci 3: 186-192.

Grant SL, Shulman Y, Tibbo P, Hampson DR, Baker GB (2006). Determination of d-serine and related neuroactive amino acids in human plasma by high-performance liquid chromatography with 
fluorimetric detection. J Chromatogr B Analyt Technol Biomed Life Sci 844: 278-282.

Hamilton NB, Attwell D (2010). Do astrocytes really exocytose neurotransmitters? Nat Rev Neurosci 11: 227-238.

Hussaini SMQ, Jun $\mathrm{H}$, Cho $\mathrm{CH}$, Kim HJ, Kim WR, Jang $\mathrm{M}-\mathrm{H}$ (2013). Heat-induced antigen retrieval: an effective method to detect and identify progenitor cell types during adult hippocampal neurogenesis. J Visualized Exp: JoVE 78: 50769.

Ishiwata S, Umino A, Balu DT, Coyle JT, Nishikawa T (2015). Neuronal serine racemase regulates extracellular D-serine levels in the adult mouse hippocampus. J Neural Transm 122: 1099-1103.

Jang MH, Bonaguidi MA, Kitabatake Y, Sun J, Song J, Kang E et al (2013). Secreted frizzled-related protein 3 regulates activitydependent adult hippocampal neurogenesis. Cell Stem Cell 12: 215-223.

Jun H, Mohammed Qasim Hussaini S, Rigby MJ, Jang MH (2012a). Functional role of adult hippocampal neurogenesis as a therapeutic strategy for mental disorders. Neural Plast 2012: 854285.

Jun H, Mohammed Qasim Hussaini S, Rigby MJ, Jang MH (2012b). Functional role of adult hippocampal neurogenesis as a therapeutic strategy for mental disorders. Neural Plast 2012: 854285.

Kang E, Wen Z, Song H, Christian KM, Ming GL (2016). Adult neurogenesis and psychiatric disorders. Cold Spring Harb Perspect Biol 8: pii: a019026.

Kanski R, van Strien ME, van Tijn P, Hol EM (2014). A star is born: new insights into the mechanism of astrogenesis. Cell Mol Life Sci 71: 433-447.

Kempermann G, Jessberger S, Steiner B, Kronenberg G (2004). Milestones of neuronal development in the adult hippocampus. Trends Neurosci 27: 447-452.

Kempermann G, Kronenberg G (2003). Depressed new neuronsadult hippocampal neurogenesis and a cellular plasticity hypothesis of major depression. Biol Psychiatry 54: 499-503.

Kheirbek MA, Klemenhagen KC, Sahay A, Hen R (2012). Neurogenesis and generalization: a new approach to stratify and treat anxiety disorders. Nat Neurosci 15: 1613-1620.

Kondziella D, Brenner E, Eyjolfsson EM, Sonnewald U (2007). How do glial-neuronal interactions fit into current neurotransmitter hypotheses of schizophrenia? Neurochem Int 50: 291-301.

Koyama Y (2015). Functional alterations of astrocytes in mental disorders: pharmacological significance as a drug target. Front Cell Neurosci 9: 261.

Kvajo M, McKellar H, Arguello PA, Drew LJ, Moore H, MacDermott $\mathrm{AB}$ et al (2008). A mutation in mouse Disc1 that models a schizophrenia risk allele leads to specific alterations in neuronal architecture and cognition. Proc Natl Acad Sci USA 105: 7076-7081.

Laroche S, Davis S, Jay TM (2000). Plasticity at hippocampal to prefrontal cortex synapses: dual roles in working memory and consolidation. Hippocampus 10: 438-446.

Ma DK, Ming GL, Song H (2005). Glial influences on neural stem cell development: cellular niches for adult neurogenesis. Curr Opin Neurobiol 15: 514-520.

Ma TM, Abazyan S, Abazyan B, Nomura J, Yang C, Seshadri S et al (2013). Pathogenic disruption of DISC1-serine racemase binding elicits schizophrenia-like behavior via D-serine depletion. $\mathrm{Mol}$ Psychiatry 18: 557-567.

Millar JK, Wilson-Annan JC, Anderson S, Christie S, Taylor MS, Semple CA et al (2000a). Disruption of two novel genes by a translocation co-segregating with schizophrenia. Hum Mol Genet 9: 1415-1423.

Millar JK, Wilson-Annan JC, Anderson S, Christie S, Taylor MS, Semple CA et al (2000b). Disruption of two novel genes by a translocation co-segregating with schizophrenia. Hum Mol Genet 9: 1415-1423.
Molofsky AV, Krencik R, Ullian EM, Tsai HH, Deneen B, Richardson WD et al (2012). Astrocytes and disease: a neurodevelopmental perspective. Genes Dev 26: 891-907.

Niwa M, Cash-Padgett T, Kubo KI, Saito A, Ishii K, Sumitomo A et al (2016). DISC1 a key molecular lead in psychiatry and neurodevelopment: no-more disrupted-in-Schizophrenia 1. Mol Psychiatry 21: 1488-1489.

Parpura V, Heneka MT, Montana V, Oliet SH, Schousboe A, Haydon PG et al (2012). Glial cells in (patho)physiology. J Neurochem 121: 4-27.

Pletnikov MV, Ayhan Y, Nikolskaia O, Xu Y, Ovanesov MV, Huang $\mathrm{H}$ et al (2008). Inducible expression of mutant human DISC1 in mice is associated with brain and behavioral abnormalities reminiscent of schizophrenia. Mol Psychiatry 13: 173-186 115.

Radzishevsky I, Sason H, Wolosker H (2013). D-serine: physiology and pathology. Curr Opin Clin Nutr Metab Care 16: 72-75.

Revest JM, Dupret D, Koehl M, Funk-Reiter C, Grosjean N, Piazza $\mathrm{PV}$ et al Adult hippocampal neurogenesis is involved in anxietyrelated behaviors. Mol Psychiatry, (2009) 14: 959-967.

Santarelli L, Saxe M, Gross C, Surget A, Battaglia F, Dulawa S et al (2003). Requirement of hippocampal neurogenesis for the behavioral effects of antidepressants. Science 301: 805-809.

Schizophrenia Working Group of the Psychiatric Genomics C (2014). Biological insights from 108 schizophrenia-associated genetic loci. Nature 511: 421-427.

Schnieder TP, Dwork AJ (2011). Searching for neuropathology: gliosis in schizophrenia. Biol Psychiatry 69: 134-139.

Schoenfeld TJ, Cameron HA (2015). Adult neurogenesis and mental illness. Neuropsychopharmacology 40: 113-128.

Snyder JS, Soumier A, Brewer M, Pickel J, Cameron HA (2011). Adult hippocampal neurogenesis buffers stress responses and depressive behaviour. Nature 476: 458-461.

Song H, Stevens CF, Gage FH (2002). Astroglia induce neurogenesis from adult neural stem cells. Nature 417: 39-44.

Sullivan PF, Daly MJ, O'Donovan M (2012). Genetic architectures of psychiatric disorders: the emerging picture and its implications. Nat Rev Genet 13: 537-551.

Sultan S, Gebara EG, Moullec K, Toni N (2013). D-serine increases adult hippocampal neurogenesis. Front Neurosci 7: 155.

Sultan S, Li L, Moss J, Petrelli F, Casse F, Gebara E et al (2015). Synaptic integration of adult-born hippocampal neurons is locally controlled by astrocytes. Neuron 88: 957-972.

van Praag $\mathrm{H}$, Schinder AF, Christie BR, Toni N, Palmer TD, Gage FH (2002). Functional neurogenesis in the adult hippocampus. Nature 415: 1030-1034.

Wang JW, David DJ, Monckton JE, Battaglia F, Hen R (2008). Chronic fluoxetine stimulates maturation and synaptic plasticity of adult-born hippocampal granule cells. J Neurosci 28: 1374-1384.

Wolosker H, Balu DT, Coyle JT (2016). The rise and fall of the d-serine-mediated gliotransmission hypothesis. Trends Neurosci 39: 712-721.

Wolosker H, Blackshaw S, Snyder SH (1999). Serine racemase: a glial enzyme synthesizing $\mathrm{D}$-serine to regulate glutamate- $\mathrm{N}$ methyl-D-aspartate neurotransmission. Proc Natl Acad Sci USA 96: 13409-13414.

Zhang Y, Chen K, Sloan SA, Bennett ML, Scholze AR, O'Keeffe S et al (2014). An RNA-sequencing transcriptome and splicing database of glia, neurons, and vascular cells of the cerebral cortex. J Neurosci 34: 11929-11947.

Zhou M, Li W, Huang S, Song J, Kim JY, Tian X et al (2013). mTOR Inhibition ameliorates cognitive and affective deficits caused by Disc1 knockdown in adult-born dentate granule neurons. Neuron 77: 647-654.

Supplementary Information accompanies the paper on the Neuropsychopharmacology website (http://www.nature.com/npp) 